\title{
Use and translation of abbreviations and acronyms in scientific texts
}

\author{
Ilya Ulitkin $^{1, *}$, Irina Filipova $^{1}$, Natalia Ivanova $^{1}$, and Yuriy Babaev ${ }^{1}$ \\ ${ }^{1}$ Moscow Region State University, 10A, Radio Str., 105005, Moscow, Russia
}

\begin{abstract}
Most new concepts both in the Russian and English languages are expressed using phrases or compound words, because such complex words make it possible to represent a particular concept with completeness and accuracy. But multicomponent terms - complex words and phrasesare cumbersome; therefore, there is a need to abbreviate them in one way or another. In some cases this leads to the use of short versions of the term in the form of only one main component, while in others, various types of abbreviations are used, which can save time. However, their imprecise or incorrect translation can change or confuse the intended meaning. The paper discusses the differences in using abbreviations and acronyms in British and American scientific texts, as well as difficulties of their translation and optimal strategies of interlanguage adaptation. The investigation is performed using various research techniques, including a comparative method, a continuous sampling method, semantic structure analysis, and contextual analysis. It is shown that the existing modern classifications of abbreviations greatly differ in linguistic scientific literature and lexical units are abbreviated using various methods. It is found that there exist various traditions of their usage in scientific and technical texts. It is demonstrated that various standards for introducing, spelling, and punctuating abbreviations and acronyms in British and American scientific journals pose additional difficulties in the work of a translator in the field of science and technology, provokes translation errors and requires the use of normalization and explication as the main strategies for their translation. The paper may be of interest for those who translate scientific texts for British and American readership.
\end{abstract}

\section{Introduction}

Over the past decades modern languages have exhibited a tendency towards 'economical use,' which consists in providing a maximum amount of data transmission per unit of time. In this regard, we currently observe an "explosive increase in the use of abbreviations" in languages [1]. This is especially obvious in the language of science and technology, which is characterized by a large number of different types of abbreviations and acronyms. The growing number of abbreviations, which find use in modern languages, is quite natural.

\footnotetext{
* Corresponding author: ulitkin-ilya@yandex.ru
} 
Abbreviations are an important component of many scientific and technical papers, and if one uses them effectively, they not only reduce space, but also facilitate the reading and understanding of a text. Translators often face complex sentences that are abundant in terms for which we already have well-established shortened forms. An important feature of modern scientific and technical language is the large number of abbreviations and acronyms. For example, the sentence 'Because X-ray computed tomography is the most common form of computed tomography in medicine and various other contexts, the term computed tomography alone is often used to refer to X-ray computed tomography, although other types exist (such as positron emission tomography and single-photon emission computed tomography). The existing methods of computed tomography and magnetic resonance tomography, including diffuse optical tomography, require a large rapid-access memory and considerable computational resources' will be easier understood by scientists if appropriate abbreviations are introduced. Of course, the advantages of such an "economical use" will be obvious, provided the abbreviations have been defined previously in the text, in accordance with the scientific writing rules, or if use is made of occasional abbreviations, in compliance with the house style of a scientific journal. When voiced, the time of their pronunciation is much shorter than the corresponding concept, and when written, 'their effect' is even more impressive: 'Because X-ray CT is the most common form of CT in medicine and various other contexts, the term CT alone is often used to refer to $X$ ray $C T$, although other types exist (such as PET and SPECT). The existing methods of CT and MRT, including DOT, require a large RAM and considerable computational resources.' From these examples we can see that if abbreviations are used in the above sentences, the text is reduced by about 1.25 times (69 words vs. 54 words). This demonstrates that abbreviations and acronyms are an important part of scientific articles and scientific and technical translation. Their proper use not only reduces space, but also facilitates the process of perception of a scientific text, which otherwise would be too long and difficult to read.

Thus, in this paper we consider the characteristic features of abbreviations and acronyms in scientific texts. The rules that we cover in this piece of writing are not necessarily a review of all possible options which one might consider, but provide practical examples we have encountered in the course of our work as linguists and translators.

\section{Research method and materials}

Methods used to study abbreviations and acronyms are determined by the objectives of this work. The comparative method is used to systematically compare full lexical units and shortened lexical units of various types in order to identify structural and semantic transformations in the process of their abbreviation. The method of continuous sampling of lexicographic publications and scientific periodicals is employed to select the empirical material and to identify publishing policies regarding the use of various (especially occasional) abbreviations. The method of semantic structure analysis of lexical units makes it possible to establish general and distinctive properties of the structure and meaning of shortened lexical units to develop their typology. The method of contextual analysis helps identify the degree of language economy (through the use of abbreviations) and reveal meaningful differences between semantically/terminologically close abbreviated units.

The research material consisted of lexical units selected from written scientific and technical sources: British and American scientific journals (American Journal of Physics, Advances in Physics, A European Journal of Chemistry, Advances in Natural Sciences, Nature, Quantum Electronics, etc.) and lexicographic resources (various dictionaries and reference materials, such as online dictionaries, Style Guides, and Manuals for Writes and 
Editors). Abbreviations from the lexicographic sources of military sublanguage were occasionally used in the analysis.

\section{Abbreviations vs. acronyms}

A variety of abbreviations and acronyms can be found in academic and professional texts. Because they are quite often registered in lexicographical sources, they can be considered lexical units of scientific and technical language. In the English language, abbreviations, according to their graphical and sound representation, are usually divided into abbreviations and acronyms.

According to the online Oxford English Dictionary [2], an abbreviation is a shortened form of a word or phrase, for example, etc. for et cetera, e.g. for exempli gratia, $k V$ for kilovolt, $W$ for Watt, $J$ for Joule, etc. In the same dictionary an acronym is an abbreviation formed from the initial letters of other words and pronounced as a single word [for example, laser (Light Amplification by Stimulated Emission of Radiation) or COIL (Chemical Oxygen-Iodine Laser)]. Despite the fact that acronyms and abbreviations are generally formed from combinations of capital letters, they can also be composed of lowercase letters, or even consist of capital and lowercase letters together, for example, $m R N A$ (messenger ribonucleic acid), ${ }_{n S O C T}$ (nano-sensitive optical coherence tomography), $d B x$ (decibels above reference coupling) or $p p b$ (parts per billion).

In his Dictionary of Linguistics and Phonetics David Crystal [3] writes that in the everyday sense the term 'abbreviation' is studied in modern linguistics as part of word formation. Crystal distinguishes several ways in which words can be shortened, namely, initialisms or alphabetisms, which reflect the separate pronunciation of the initial letters of the constituent words [for example, $T V$ (television), $L N$ (liquid nitrogen), $A A$ (antenna array), etc.]; acronyms, which are pronounced as single words [for example, radar (radio detection and ranging)]; clipped forms or clippings, which are reductions of longer forms, usually removing the end of the word [ad (advertisement), lab (laboratory), exam (examination), etc.], but sometimes the beginning [plane (airplane), phone (telephone), etc.], or both beginning and ending together [flu (influenza)]; and blends, which combine parts of two words [transceiver (transmitter + receiver), informatics (information + electronics), bit (binary + digit), breathalyzer (breath + analyzer), etc.].

In addition, this classification can be also supplemented by abbreviations formed by a combination of the initial letter of a word with an unabbreviated word such as H-bomb (hydrogen bomb), e-mail (electronic mail), etc. (for example, electron-beam lithography (often abbreviated as e-beam lithography) is the practice of scanning a focused beam of electrons to draw custom shapes on a surface covered with an electron sensitive film) and abbreviations formed by a combination of a clipped form of a word with an unabbreviated word such as nano-object (nanometer object), bio-tissue (biological tissue), etc. (for example, This raises a number of problems associated with introduction of bio-materials directly into the body: disruption of homeostasis, implant rejection and its poor integration). We should not also forget about alphanumeric abbreviations, which are now widely used in scientific and technical texts ( $3 D$ - three-dimensional), and 'extremely' often in the language of today's youth in the Internet communication (CUL8R - see you later, $4 U-$ for you).

According to Nelyubin [4], an abbreviation (the Italian word 'abbreviatura' comes from the Latin word 'brevis', which means 'short') is a word composed of the initial letters or initial sounds of words constituting the original phrase. Thus, an abbreviation is the result of optimization of a message, stemming from the removal of various types of "noises" from communication and making the message appropriate and short for delivering information. The abbreviation process consists in the reduction of a number of elements of 
a lexical unit and in the formation of a new one. As a result of shortening, there appears an abbreviated lexical unit, which is structurally different from the original version.

Note that an acronym is a shortened form of a word coinciding in its phonetic structure with a common word and pronounced as a single word.

In the Russian linguistic literature there exist a variety of approaches to the classification of abbreviations. For example, following the Dictionary of Linguistic Terms [5], we can classify shortened forms as follows: 1) abbreviations; 2) graphical shortenings; and 3) clippings. In turn, each of them is subdivided into other types.

The first type of abridgements, i.e. abbreviations, is mainly represented by initialisms, which are subdivided into a) alphabetisms [for example, $d c$ (direct current)]; b) syllabic abbreviations consisting mainly of the initial syllables of several words [VoIP (voice over Internet protocol)]; c) acronyms, which is actually a kind of an abbreviation formed from the initial letters of words or phrases [CATV (cable TV), The design and function of a scanning electron microscope (SEM) is very similar to an electron-probe micro-analyzer (EPMA) and considerable overlap in capabilities exists between the two instruments].

The second type of contraction, i.e. graphical shortenings, consists in graphic reduction, when the clipped part of the words is substituted by a dot [Vol. (volume), $p$. (page)], or a hyphen replacing the words clipped from the middle $[D D$ (deuterium-deuterium)], or a slash [DIOB (digital input/output buffer)]. It should be emphasized that graphic shortenings are found only in the written language, while in speech, their relevant full forms are used [6].

The third type of shortened words, i.e. clippings, has become especially popular and widespread in English over the past decade. Linguists distinguish several types of clipping: 1) apocope, i.e. the final part of the word is removed [for example, rev (revolution)]; 2) apheresis, i.e. the initial part of the word is removed [for example, jams (pyjamas)]; 3) syncope, i.e. the middle part of the word is omitted [for example, telecoms (telecommunications)]; and 4) mixed-type clipping, i.e. a combination of basic types [for example, comp (accompaniment)] [7].

Thus, we can conclude that in modern linguistics there exist multiple classifications of abbreviations. Nevertheless, they can be mainly divided into abbreviations and acronyms, blends, graphic shortenings and clippings. It should be noted, however, that between these types of shortened words there are a number of border phenomena in which the shortening, after certain phonetic and morphological changes, undergoes a transition from one type to another.

Of greatest interest to us are abbreviations and acronyms because blends, clippings and graphical shortenings do not seem difficult to use and translate. Abbreviations and acronyms, despite the fact that both are formed from the initial letters of a phrase, have one important difference: abbreviations may not be pronounced as a single word. This fact causes the difference in the use of abbreviations and acronyms in language and speech. It is also important that in some cases it is impossible to determine whether the shortened word is an acronym or an abbreviation and whether this or that phrase can be shortened. For example, Wikipedia [8] recommends using abbreviations WWII or WW2 for the Second World War, while the Stylebook and Briefing on Media Law [9] and the Chicago Manual of Style [10] do not recommend the use of abbreviations in this case. For example, an abbreviation is sometimes formed from an initial sound rather than from an initial letter of the terminological phrases (such as $X$ in $X M L$, extensible markup language), or from the application of a number ( $W 3 C$, World Wide Web Consortium). In addition, acronyms and abbreviationы are sometimes combined into a single word (JPEG, the first letter is spelled out and the subsequent letters form the acronym). Sometimes it is impossible to distinguish between abbreviations and acronyms ( $F A Q$, for example, can be pronounced as a word and spelled out). 
The above explanation allows us to conclude that abbreviations are any shortened forms of the word, and acronyms are abbreviations pronounced as words [for example, AIDS (acquired immune deficiency syndrome)]. Of course, a scientist writing a scientific or technical paper probably does not need to know the difference between these two concepts, but for linguists and translators this misunderstanding and ignorance may lead to misuse of terms and inadequate translation.

\section{Use of abbreviations and acronyms in scientific and technical texts}

Various style guides give many recommendations on the use of abbreviations and acronyms in the texts. Using the most respected manuals of style - the Chicago Manual of Style [10], the Oxford Guide to Style [11], the Publication Manual of American Psychological Association [12], the MLA Handbook for Writers of Research Papers and the MLA Style Manual and Guide to Scholarly Publishing [13], etc.- we have made an attempt to summarize the experience of various publishers and present here the most common uses of abbreviations and acronyms.

1) The terms should be spelled out on their first occurrence and only then followed by the abbreviation in parentheses. For example,

Stimulated Brillouin scattering (SBS) is frequently encountered when narrow-band optical signals are amplified in a fiber amplifier, or just propagated through a passive fiber.

Nevertheless, other methods of introducing an abbreviation/acronym into a text are possible. For example,

The National Aeronautics and Space Administration was founded in 1958. Since its inception, NASA has...

However, TLAs (tunable laser assemblies) feature optional SBS suppression implemented through a controlled frequency dither.

Standard abbreviations (for example, units of measurement, or states and counties) do not need to be defined. For example,

Here, $E$ is the energy measured in $\mu J$.

Some publishers do not require decoding abbreviations and acronyms that are registered in dictionaries [14] without an abbreviation label. Nevertheless, most scientific journals have their own house styles according to which the use of less familiar abbreviations should be limited to those terms that occur frequently enough to warrant abbreviation - roughly five times or more within an article or chapter - and the terms must be spelled out on their first occurrence.

2) With abbreviations and acronyms that appear in capital letters (and even with those that contain lowercase letters), we should not use spaces and periods (CEO, DEMUX, $P h D)$. An exception is the abbreviation of the United States, U.S. (this is especially typical of American English rather than of standard British English), used as an adjective (for example, U.S. companies) and such abbreviations as R.S.V.P., N.B. and R.I.P. Periods in abbreviations are used to abbreviate Latinisms (etc., etcetera; p.m., post meridiem; and et $a l$., et alia), initials standing for given names (J. F. Kennedy, we should note that initials are followed by a period and a space; neither periods nor spaces are used when an entire name is replaced by initials, such as, JFK airport), information in references (Vol., volume; $p$., page; $p p$., pages; and 4th ed., 4th edition), academic degrees and titles (for example, $D r$., Prof., Esq.) and words ending in a lowercase letter (no. as number, nos. as numbers). In addition, the references often make use of abbreviated names of journals, such as: Quantum Electron., Zh. Eksp. Teor. Fiz., J. Exp. Theor. Phys., etc. Nevertheless, it is worth 
mentioning that the correct form of the abbreviation can usually be found on the publisher's site or in special lists, for example, ISI Journal Title Abbreviations [15].

Contraction of civil and military ranks is only possible if they are followed by name and surname. Otherwise, the title should be written in full. For example, Rep. Dan Lipinski, Representative Lipinski; Sen. Kirsten E. Gillibrand, Senator Gillibrand; and Rear Adm. Carol M. Pottenger, Rear Admiral Pottenger.

It is worth noting that, unlike American English, in standard British English periods are often omitted in academic degrees and titles (for example, $\mathrm{Dr}, \mathrm{Mr}, \mathrm{Mrs}, \mathrm{Esq}$ ). Also, periods may be replaced by the slash, which means per (for example, $c / o$ or $n / a$ ).

The units of measurement used in non-scientific style are usually written in full, while scientific and technical texts make use of abbreviations without periods (for example, $f t, L$, $c m, n s)$. The exception is the contraction of the word inch (in.), which is done in order to avoid confusion with the preposition in. Units are reduced only in case when numerical values are used, for example, $7 \mathrm{mg}, 12 \mathrm{~km}, 120 \mathrm{~W}$, etc., although here, much depends on the policies of the journal. Abbreviations for units can be found in the SI brochure [16].

3) Depending on the situation, an abbreviation can be preceded by an indefinite, definite or zero article. The choice of $a$ or $a n$ is determined by the way the abbreviation is read aloud. For example, an HMO, a UFO, a NATO member, a LOOM parade, an AA meeting, a AA battery (pronounced "double A"), an NAACP convention, an NBA coach, an HIV test, an MS symptom (a symptom of multiple sclerosis), but a MS by ... (would be read as "a manuscript by ...").

Acronyms that are read as words are rarely preceded by the articles $a$, an, or the (for example, scientists of NASA). Exceptions are grammatical constructions in which an acronym is used adjectivally (for example, a NASA initiative).

4) Abbreviations with an ampersand (\&) have no spaces on either side, for example: This $R \& D$ Group combines leading Russian $3 D$ visualization companies united to create and develop tools for $3 D$ visualization industry.

Extremely interesting is the use of abbreviations etc. and et al. in text. The abbreviation etc. (et cetera, literally "and others of the same kind") is preceded and (if it does not end the sentence) followed by a comma if it is the final item in a series. Such equivalents as and so forth and and the like are generally used in the same manner. (In official documents etc. should be avoided, although, as a rule, this abbreviation is allowed in lists and tables as well as in notes and parenthesis.)

Proper technique (with attention paid to posture, wrist position, distance from the keyboard, etc., in the later performances) is not in and of itself sufficient to explain Rubinstein's endurance.

The philosopher's population studies, classic textbooks, stray notes, and so forth, were found in the attic.

The abbreviation et al. (et alia [neuter], et alii [masculine] or et aliae [feminine], literally "and others") is most often used in bibliographic references. If et al. follows immediately a single item, a comma is not needed (for example, Smith et al.). If et al. follows several items, it is preceded by a comma (for example, Smith, Jones, et al.).

Of interest are also abbreviations e.g. (exempli gratia) and i.e. (id est). In standard British English they are not followed by a comma, while in American English these abbreviations are surrounded by commas. For example, hand tools, e.g. hammer and screwdriver and hand tools, i.e. those able to be held in the user's hands (British English), and hand tools, e.g., hammer and screwdriver and hand tools, i.e., those able to be held in the user's hands (American English).

Thus, abbreviations of various types penetrate into all layers of the lexicon of modern European languages. They accumulate information in the linguistic sign for its faster transfer and assimilation. In addition, they increase the effectiveness of communication, 
reflect the tendency towards 'economical use' of the language, supplement and enrich its vocabulary, as well as make it entertaining and interesting. In scientific and technical texts, in accordance with the tradition of the journal, occasional abbreviations help create a vertical context. After the first mention (after the full term), they take the function of a symbol, which is further used in the text. Moreover, they act as graphical visualizers of new terms and attract the reader's attention to the corresponding sections of the text, as well as serve as markers of the information space of the text and organize text architectonics. At the same time, the abbreviation should obey, on a general basis, the rules for translating vocabulary, which are based on semantic correspondences between the correlative units of a given pair of languages. Proximity to terms, i.e. absence of connotations, even more emphasizes the need to convey the semantic meaning of abbreviations.

\section{Plurals of abbreviations and acronyms}

Different dictionaries and style guides treat the plural forms of abbreviations and acronyms differently. For example, the Merriam-Webster English Dictionary [14] describes two ways of forming the plurals: plurals of abbreviations consisting of uppercase letters are formed by adding the ending $s$, for example: COIL $\rightarrow$ COILs, URL $\rightarrow U R L s$, etc. Abbreviations derived from lowercase letters, or abbreviations with periods form plural forms by using the apostrophe and ending $s$. For example, dot your i's and cross your t's.

The Chicago Manual of Style [10] recommends adding an apostrophe plus $s$ to the end if the abbreviation has more than one period, for example, G.I. $\rightarrow$ G.I.'s. However, this variant is the least preferred.

The Oxford Guide to Style [11] writes that the plurals of abbreviations should not be formed by using an apostrophe plus $s$ in the following cases: (i) after numbers, for example, The interest in this field of science has significantly increased since 1980s; and (ii) after abbreviations that consist of capital letters, for example, SOSs, FPRs (Fabry-Perot resonators). Nevertheless, the apostrophe is used in the formation of plural abbreviations consisting of lowercase letters or abbreviations with periods inside abbreviations, for example, Ph.D.'s, do's and don'ts.

The Webster Guide to Grammar and Writing [17] also shares the point of view of the Oxford Guide to Style with the only difference that it is preferable to use an apostrophe, if an abbreviation or acronym ends in $S$, for example, $S O S \rightarrow S O S^{\prime} s$ or Superfluorescent fiber sources (SFS's) find wide application in many areas of science and technology: in fiberoptic gyroscopes, ophthalmology, optical time-domain reflectometry, optical telecommunications and devices that need a signal with low temporal coherence and high spatial coherence.

Quirk, Greenbaum, et al. [18] mention both variants of forming the plural, indicating that today the plurals of abbreviations are most often formed without an apostrophe; the exceptions are plural abbreviations formed from lowercase letters, abbreviations and acronyms with periods consisting of uppercase and lowercase letters, for example, cod's; two KotOR's.

The American Heritage Book of English Usage [19] and Claremont Dictionary of English Usage [20] also support this point of view (for example, the three Rs, $x$ 's and $y^{\prime} s$, the 1990s, IRAs, URLs, BSs, MAs, PhDs, vols., Eds.). However, they mention several exceptions such as $p$. (page), pp. (pages), n. (note), nn. (notes), MS (manuscript), MSS (manuscripts).

In addition to this, all the reference books indicate that the abbreviations used to denote units do not form a plural, for example, $1.0 \mu \mathrm{g} / \mathrm{mL}, 25 \mathrm{~W} / \mathrm{cm}^{2}$. 
As can be seen, the main difference in viewpoints consists in whether to use an apostrophe or not. Nevertheless, most style guides agree that it can be used, but not necessarily.

Nevertheless, we should note more complex cases. Often an abbreviation is formed so that it is initially used in the plural, as is the case with the United Nations Organization $(U N O)$. Over time the term has been reduced to United Nations, which has a commonly accepted abbreviation $U N$ (without a plural form). Also in scientific texts we can come across terminological phrases where one of the items is plural; nevertheless, abbreviations from these phrases are not plural in form, for example: parts per billion $\rightarrow p p b$.

For example, The concentration $C$ in parts-per million (ppm) is equal to the concentration $C$ in $\mathrm{mg} / \mathrm{kg}$ and equal to 1000 times the concentration $C$ in $\mathrm{mg} / \mathrm{L}$, divided by the solution density $\rho$ in kilograms per cubic meter $\left(\mathrm{kg} / \mathrm{m}^{3}\right)$ :

$$
\left.C_{(\mathrm{ppm})}=C_{(\mathrm{mg} / \mathrm{kg})}=1000 \times C_{(\mathrm{mg} / \mathrm{L})} / \rho_{(\mathrm{kg} / \mathrm{m}}{ }^{3}\right)
$$

In water solution, the concentration $C$ in ppm is equal to 1000 times the concentration $C$ in $m g / L$ divided by the water solution density at temperature of $20^{\circ} \mathrm{C}, 998.2071 \mathrm{in} \mathrm{kg} / \mathrm{m}^{3}$ and approximately equal to the concentration $C$ in $\mathrm{mg} / \mathrm{L}$ :

$$
C_{(\mathrm{ppm})}=1000 \times C_{(\mathrm{mg} / \mathrm{L})} / 998.2071_{(\mathrm{kg} / \mathrm{m})} 3 \approx 1_{(\mathrm{L} / \mathrm{kg})} \times C_{(\mathrm{mg} / \mathrm{L})}
$$

Nevertheless, it is possible to come across (especially in the military-technical literature) examples of the use of the plural for such abbreviations. Their plurals are formed by adding $s$ at the end of the abbreviation, regardless of how the plural of the full form is formed: cf. $P O W$ (prisoner of war) - POWs (prisoners of war); OD (order of the day) - ODs (orders of the day); PFC (private first class) - PFCs (privates first class); EM (enlisted man) - EMs (enlisted men); Sig (signalman) - sigs (signalmen).

Thus, Nelyubin et al. [21] indicate that it is essential for the translation that abbreviations may perform various syntactic functions in accordance with the rules of English grammar, i.e. have plural forms, show the possessive case and the past tense, and use affixes and articles. All this often makes their understanding and translation difficult. But the special difficulty is that the meaning of abbreviations, which undergo certain semantic shifts, often do not coincide with the meaning of terms in their full form. For example, the terms Military Police and Women's Army Corps are the names of certain organizations, and the abbreviations for these terms may refer to the member of this organization: an $M P$ is a military police officer, and $a W A C$ is a servicewoman of the women's auxiliary service of the ground forces; the term radio detection and ranging denotes an action, i.e. detection and determination of distances by radio signals, whereas its abbreviation is a device by which this action is performed, radar is a radio detection and ranging device.

The above examples show that, in most cases, abbreviations form plurals according to the general rule, i.e. take the ending $s$. Some abbreviations form plurals by doubling the final letter. However, uniformity in the graphic representation of the abbreviations is still absent at the moment. The formation of abbreviations, as a result of which a complex conglomerate (an AA meeting, a AA battery) emerges on the basis of shortened lexical phrases, serves as a source of homonymy and syncretism, which prevents the differentiation of terms until a certain stage in the development of scientific thought. Thus, these peculiarities of the derivation and functioning of abbreviations pose certain difficulties in their translation. 


\section{Translation strategies}

The above-discussed standards of British and American linguistic cultures and of individual publishing houses are not the only difficulty when it comes to translation of abbreviations from one language into another. Being an extremely complex process, translation aims at reaching a fragile balance between the equivalence of a source text and the used translation strategies. Yeltsov [22] notes that translators experience comparable (almost equal) difficulties in translating abbreviations from their native language into a foreign one and from a foreign language into their native one. Let us try to list the main causes of difficulties in the interlanguage adaptation of abbreviations.

1. The use of abbreviations in scientific discourse, especially in periodicals, is associated with the presence of a common terminological base (research experience) and personal experience (skills to present scientific results in print) at the author's and the reader's levels. In the case of a significant discrepancy in background knowledge and skills, the translation of an abbreviation, which is a substitute for an explicit expression of a multicomponent term, can lead to mistakes. At the stage of perception of an original abbreviation, a translator can encounter difficulties in the identification of the abbreviation and the problems of its correlation with the full terminological unit.

2. Polysemy of abbreviations creates additional difficulties in recognizing their semantic content. Nelyubin [23] notes that "some of them can have several dozen registered definitions in dictionaries. For example, the abbreviation $A$ in English has more than 60 different definitions; $B$, more than 40 ; and $C A$, more than 20, etc".

3. Multiple standards discussed above also create prerequisites for cognitive errors. In this case, different (non-identical) traditions of scientific journals are closely intertwined with the linguistic features of their formation and functioning. To a large extent, the difficulties in understanding abbreviations are related to the fact that they can be indicative of various parts of speech and express different syntactic functions. Thus, the ending $s$ can be assigned to the plural form of an abbreviation (for example, cod's), the possessive case (for example, Co's employees), the shortened third-person singular verb in the present simple tense (for example, The Zavala family thxs the Marshal group...).

4. In the course of adaptation, the perceptual and cognitive problem solving is transformed into a translation one. At the recoding stage, the variability of translation correspondences comes into conflict with the requirement of a single meaning of the term and the unambiguous equivalence of its translation into the target language. The translator must not only translate the original message, taking into account the interlanguage correspondence of the abbreviation, but also follow the publishing practice of a particular journal; otherwise, the irrelevance of the translation solution will be obvious to readers, and they will consider it as a 'marker of the text's non-authenticity.' Then, an explicit translation error can be treated as a failure of proper communication.

5. Abbreviations described above (their syncretism and homonymy stemming from the formation and variability in connection with the linguistic and cultural traditions of the British and American scientific literature) form a linguistic level of translation problems. Syncretism and homonymy of abbreviations play the role of destructive interference at the level of word formation in this case. Previously, this type of interference (word-building) was not described in the literature, but in view of its obviousness, we consider it possible to briefly note that it occupies an intermediate position between lexical and morphological levels and is the result of asymmetry of language resources and the asynchrony of the formation of terminology and the course of derivational processes in the source and target languages [24].

The translation strategies of abbreviations in various languages have been repeatedly studied and revealed a generally universal nature of their translation (see, e.g., [23-26] and 
references therein). The general and axiomatic principle of abbreviation translation is the necessity to reach a balance of equivalence of abbreviations in the source language and the target language. This principle can be implemented using several translation strategies.

- Replacement of an abridgement of a source language with an equivalent abridgement of a target language. In searching for an equivalent abridgement the translator finds some reference source (dictionary, microcontext, etc.) and uses the available abbreviation of the target language.

- Adoption of the abbreviation of a source language (in translating from English into Russian). This strategy is used to translate alphabetic-numeric abbreviations, which are part of some indexing systems, specifications, etc. Abbreviated names of English brands of machines, apparatuses, devices, etc., are usually adopted in the original spelling.

- Translation of abbreviations by means of the transliteration method. Apart from technical and scientific translation, this strategy is widely employed to adopt names of some military alliances, industrial companies, and political organizations from a source language into a target language, with the abridgements being usually written in capital letters and without quotes.

- Translation of abbreviations by means of the transcription method.

- Descriptive translation of an abbreviation. This strategy of abridgement translation corresponds to translation of the root of the abbreviation with the microcontext taken into account. This technique is used when it is difficult or impossible to use the previously described strategies, which is often the case in the absence of abridgements in dictionaries.

- Derivation of a new abridgement. This strategy can be recommended only when the translator closely collaborates with specialists and has good reasons to rule out all above-mentioned strategies of abbreviation translation. This newly derived abridgement should be approved by specialists.

Thus, the translation strategies presented above can be divided into two large groups, depending on the strategy in question, i.e. normalization and explicitation.

In Russian-language translation studies, normalization, i.e. correspondence of the target text to the literary norms and the linguistic and cultural tradition of the target language, does not have the status of a special strategy, but is one of the necessary requirements for a translated text [4]. At the same time, European and American scholars consider normalization (sometimes referred to as standardization) to be a universal translation tool, i.e. "a special characteristic that is inherent in target languages rather than in source languages and is simultaneously independent of a specific language system" [27].

Explicitation $[28,29]$ is a more detailed and voluminous presentation of the original information in the target language, which is due to the desire of the translator to compensate for the differences in the source and target languages and cultures. This strategy also reflects the general tendency of translation practice, i.e. to fully present information implicitly presented in the source text [30] and to generate texts that are more explicit than the original ones [31]. In the scientific and technical texts, this strategy finds its way due to the above-described and often closely related reasons.

Thus, translation of abbreviations is a complex process that works on various linguistic levels, such as cognitive, linguistic, and cultural. The high requirements for translation quality and the growing volume of scientific communication require that the translator not only knows well the subject (area of knowledge), but is also well aware of the translation, lexicographic, and lingua-cultural tradition and is ready for the continuous expansion of the bank of translation correspondences of abbreviations and mastering new horizons to optimize intercultural dialogue in the field of science and technology. 


\section{Conclusion}

Nowadays the language of scientific and technical literature both in Russian and English often makes use of a large number of abbreviations and acronyms. Abridgement as a trend has become quite popular and common in many languages. The development of science and technology and international integration processes give rise to the appearance of new concepts which are expressed with the help of phrases and compound words. However, such phrases and compound words can be quite cumbersome, which leads to their abridgement. The use of abbreviations and acronyms in national languages is often justified because abridgements can save time, but their inadequate translation or use can be a cause of an error in translation. Abbreviations invade the everyday speech of certain social groups and are registered in various specialized dictionaries, including electronic sources. This makes it possible not only to study but also to analyze the course of the abbreviated word formation by native speakers. Thus, in this paper, we have discussed some important points regarding the use of abbreviations in scientific journals. In addition, we have provided guidance for using abbreviations in general and scholarly writing. Therefore, the topic of research can be interesting not only for linguists but also for a wider readership, including those who translate scientific texts for American and British readership.

The authors wish to thank their colleague Stephen Garratt (England) for his helpful suggestions on manuscript editing and polishing the language of the paper.

\section{References}

1. S.S. Barbazeva, A.A. Avramenko, Bulletin of the Samara Scientific Center of the Russian Academy of Sciences 13(2), 911 (2001)

2. Oxford http://www.oxforddictionaries.com/definition/american_english/abbreviation

3. D. Crystal, The Dictionary of Linguistics and Phonetics (Oxford, Blackwell Publishing, 2008)

4. L.L. Nelyubin, Explanatory Dictionary of Translation Terms (Moscow, Flinta, Nauka Publ., 2003)

5. Dictionary of Linguistic Terms, http://dic.academic.ru/contents.nsf/lingvistic/

6. A.M. Peshkovskii, Russian Syntax from The Point of View of Science (Moscow, Yazyki slavyanskoi kul’tury Publ., 2001)

7. Abridgements in English Press, http://www.bibliofond.ru/view.aspx?id=600341

8. Wikipedia, http://en.wikipedia.org/wiki/World_War_II

9. D. Christian, S. Jacobsen, D. Minthorn, Stylebook and Briefing on Media Law (New York, Basic Books, 2011)

10. The Chicago Manual of Style (16th Edn) (Chicago, University of Chicago Press, 2010)

11. R.M. Ritter, The Oxford Guide to Style (Oxford, Oxford University Press, 2002)

12. Publication Manual of American Psychological Association (Washington, DC, American Psychological Association, 2010)

13. The MLA Handbook for Writers of Research Papers and the MLA Style Manual and Guide to Scholarly Publishing, http://www.mla.org/style_faq1

14. Merriam-Webster's Collegiate Dictionary (Massachusetts, Springfield, MerriamWebster, 2003)

15. ISI Journal Title Abbreviations, http://library.caltech.edu/reference/abbreviations/ 
16. SI brochure (8th edition - 2006, updated in 2014) http://www.bipm.org/en/si/si_brochure/

17. Guide to Grammar and Writing, http://grammar.ccc.commnet.edu/grammar/

18. R. Quirk, S. Greenbaum, G. Leech, J. Svartvik, University Grammar of English Text (Moscow, Vysshaya shkola, 1982)

19. The American Heritage Book of English Usage (Boston, Houghton Mifflin, 1996)

20. Claremont Dictionary of English Usage (Collana, Godfrey Cave Associates Ltd, 1995)

21. L.L. Nelyubin, A.A. Dormidontov, A.A. Vasil'chenko, Textbook for Developing Military Translation Skills. English Language (Moscow, Military Publishing, 1981)

22. K.A. Yeltsov, Abbreviation Translation Strategies, PhD Thesis (Moscow, 2005)

23. L.L. Nelyubin, Introduction Translation Technique (Moscow, MGOU Publ., 2007)

24. V.V. Alimov, By The Example of Professionally Oriented Intercultural Communication and Translation in The Field of Professional Communication). Doctoral Thesis (Moscow, 2004)

25. E.N. Ozhogin, Abbreviations in the Military Sublanguage (Moscow, Voennyi Universitet Publ., 1999)

26. Ya.I. Retsker, Translation Theory and Translation Practice (Moscow, Mezhdunarodnye otnosheniya Pbl., 1974)

27. M. Baker, G. Francis, E. Tognini-Bonelli, Corpus Linguistics and Translation Studies. Implications and Applications. Text and Technology (Amsterdam, Philadelphia, John Benjamins, 1993)

28. J.-P. Vinay, J. Dalbernet, La Stylistique comparée du francais et de l'anglais. Méthode de traduction (Paris, Didier, 1958)

29. K. Klaudy, Explicitation. Routledge Encyclopedia of Translation Studies (London-New York, Routledge's, 1998)

30. G. Toury, In search of a Theory of Translation (Tel Aviv, University Tel Aviv, 1980)

31. A. Chesterman, The Memes of Translation. The Spread of Ideas in Translation Theory (Philadelphia, Amsterdam, John Benjamins, 1997) 